Proyecciones Journal of Mathematics

Vol. 40, No 5, pp. 1211-1225, October 2021.

dol 10.22199 / issn. $0717-6279-4580$

Universidad Católica del Norte

Antofagasta - Chile

\title{
Extended study of biological networks using graph theory
}

\author{
Tomáš Vetrik \\ University of the Free State, South Africa \\ Received: November 2020. Accepted: March 2021
}

\begin{abstract}
We represent biological networks by a function that maps the structure of a network to a number called topological index. Topological indices have been studied for biological networks in which a person transmits a virus to two other people, and a person having the virus is in contact with exactly one other person who got the virus from someone else. We extend research in this area by studying biological networks in which a person transmits a virus to $n$ other people, where $n \geq 2$, and a person having the virus is in contact with $p$ other people $(0 \leq p \leq n-2)$ who got the virus from some other person.
\end{abstract}

Keywords: Topological index, virus, biological network.

Mathematics Subject Classification: 05C09, 05C90. 


\section{Introduction}

Topological indices measure physical, chemical and biological properties. We represent biological networks by a function that maps the structure of a network to a number called topological index.

Biological networks $H T(h)$ and $S T_{h}^{1}$ were studied by Imran et al. [7] and Gao et al. [4]; see Figures 1 and 2. They assume that a virus or bacteria is transmitted from one person to two other people, and a person having the virus is in contact with exactly one other person who got the virus from someone else. We extend research in this area by studying biological networks in which a virus or bacteria is transmitted from one person to $n$ other people, where $n \geq 2$, and a person having the virus is in contact with $p$ other people $(0 \leq p \leq n-2)$ who got the virus from someone else.

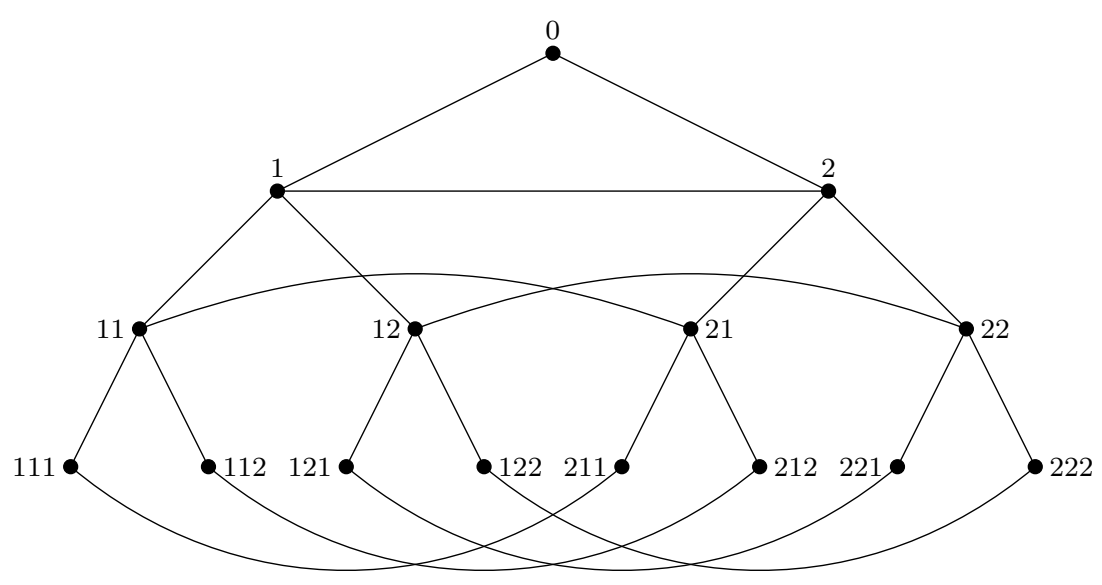

Figure 1: Network $H T(h)$ for $h=3$. 


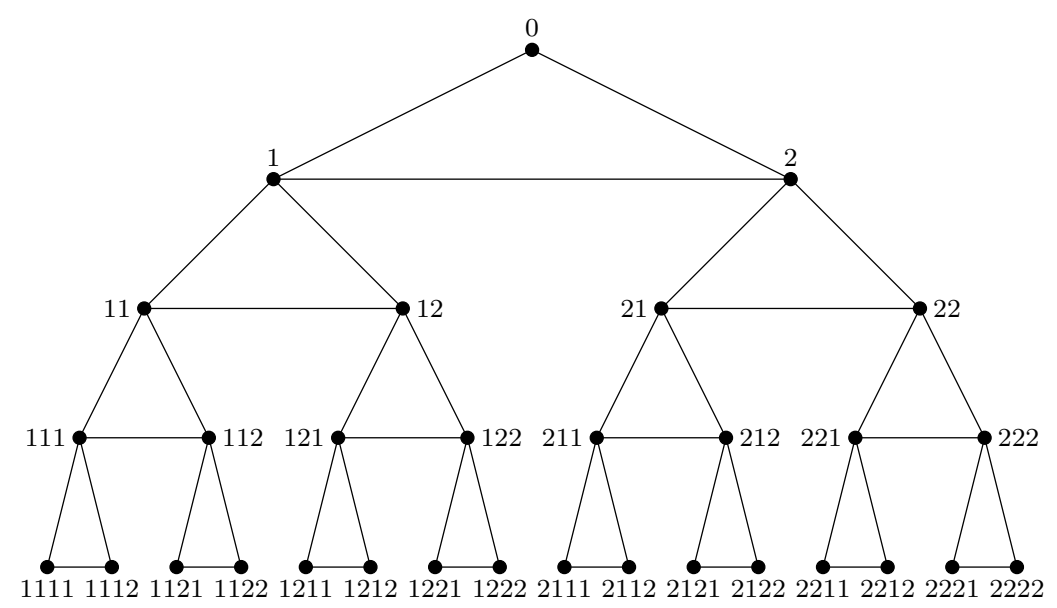

Figure 2: Network $S T_{h}^{1}$ for $h=3$.

Let $V(N)$ be the vertex set and $E(N)$ be the edge set of a network $N$. The number of edges in a shortest path between two vertices is called the distance between those two vertices. The distance between $u$ and a vertex farthest from $u$ is called the eccentricity $e c c_{N}(u)$ of a vertex $u$ in $N$.

We define complete $n$-ary trees $T_{n, h}$ of given height $h \geq 1$. Those tress are used to define our extended biological networks. The vertices of $T_{n, h}$ are partitioned into $h+1$ sets $S_{0}, S_{1}, \ldots, S_{h}$. For $i=1,2, \ldots, h$,

$$
\mathrm{S}_{i}=\left\{\left(v_{1}, v_{2}, \ldots, v_{i}\right) \mid 1 \leq v_{j} \leq n ; j=1,2, \ldots, i\right\} .
$$

A vertex $\left(v_{1}, v_{2}, \ldots, v_{i}\right)$ is sometimes denoted by $v_{1} v_{2} \ldots v_{i}$. The root 0 is the only vertex of $S_{0}$ and it is joined to the $n$ vertices of $S_{1}$. For $i=1,2, \ldots, h-1$, Each vertex $\left(v_{1}, v_{2}, \ldots, v_{i}\right)$ of $S_{i}$ is joined to

$$
\left(\mathrm{v}_{1}, v_{2}, \ldots, v_{i}, 1\right),\left(v_{1}, v_{2}, \ldots, v_{i}, 2\right), \ldots,\left(v_{1}, v_{2}, \ldots, v_{i}, n\right)
$$

of $S_{i+1}$.

For $p=0,1,2, \ldots, n-2$, the set $T_{n, h}^{p}$ includes the networks created from $T_{n, h}$ by joining every vertex $v$ in $S_{i}$ (where $i=2,3, \ldots, h$ ) to any $p$ vertices in $S_{i}$ which have the first entry equal to the first entry of $v$, and by joining every vertex in $S_{1}$ to any $p$ vertices in $S_{1}$. Note that this construction is possible only if at least one of the integers $p$ and $n$ is even. So, the set $T_{n, h}^{p}$ is empty if both $p$ and $n$ are odd. One network of $T_{n, h}^{p}$ for $p=2, n=5$ 
and $h=2$ is given in Figure 3 .

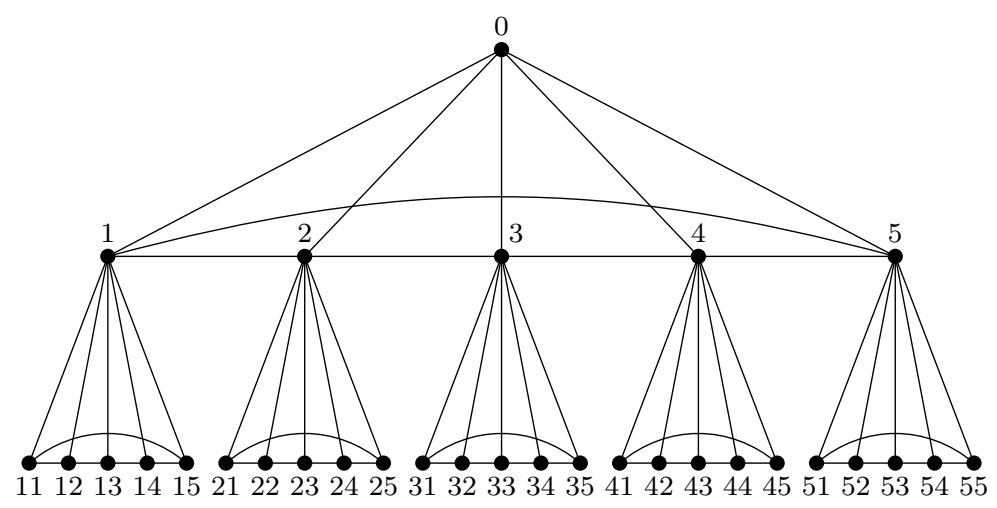

Figure 3: Network in $T_{n, h}^{p}$ for $p=2, n=5$ and $h=2$.

For any network $N \in T_{n, h}^{p}$, a vertex in $V(N) \backslash\left(S_{0} \cup S_{1}\right)$ having the first entry $j$ is not joined to a vertex having the first entry $l$, where $j \neq l$. Thus, for $i=1,2, \ldots, h$, the eccentricity of $\left(v_{1}, v_{2}, \ldots, v_{i}\right) \in S_{i}$ is $h+i$. Any vertex $\left(u_{1}, u_{2}, \ldots, u_{h}\right) \in S_{h}$, where $\left(u_{1}\right)$ is not adjacent to $\left(v_{1}\right)$ is a farthest vertex from $\left(v_{1}, v_{2}, \ldots, v_{i}\right)$.

The path

$\left(v_{1}, v_{2}, \ldots, v_{i}\right),\left(v_{1}, v_{2}, \ldots, v_{i-1}\right), \ldots,\left(v_{1}\right), 0,\left(u_{1}\right),\left(u_{1}, u_{2}\right), \ldots,\left(u_{1}, u_{2}, \ldots, u_{h}\right)$

is the shortest path connecting $\left(v_{1}, v_{2}, \ldots, v_{i}\right)$ and $\left(u_{1}, u_{2}, \ldots, u_{h}\right)$. Note that the eccentricity of 0 is $h$ in $T_{n, h}^{p}$,

The first Zagreb eccentricity index of a network $N$,

$$
\xi_{1}(N)=\sum_{u \in V(N)}\left(e c c_{N}(u)\right)^{2}
$$

and the second Zagreb eccentricity index

$$
\xi_{2}(N)=\sum_{u v \in E(N)} \operatorname{ecc}_{N}(u) e c c_{N}(v)
$$

were defined in [11]. The $\xi_{1}^{\prime}$ index

$$
\xi_{1}^{\prime}(N)=\sum_{u v \in E(N)}\left(e c c_{N}(u)+e c c_{N}(v)\right)
$$

was defined in [5]. The modified Zagreb eccentricity index is the name which will be used for the $\xi_{1}^{\prime}$ index. 
For $a \in \mathbf{R}$, we define the general eccentricity index

$$
E I_{a}(N)=\sum_{u \in V(N)}\left(e c c_{N}(u)\right)^{a} .
$$

The total eccentricity index

$$
E I_{1}(N)=\sum_{u \in V(N)} e c c_{N}(u)
$$

is obtained when using $a=1$ and the first Zagreb eccentricity index $E I_{2}=$ $\xi_{1}$ is obtained when using $a=2$.

Zagreb eccentricity indices have been investigated due to their wide applications. Eccentricity based indices of honeycomb networks were studied in [6], nanostar dendrimers in [3] and oxid networks in [8], For related works, see [1], [2], [9] and [10].

\section{Results}

Let $n \geq 2$ and $h \geq 1$ be positive integers. In our main results we often use $\sum_{i=c}^{h} n^{i}, \sum_{i=c}^{h} i n^{i}$ and $\sum_{i=c}^{h} i^{2} n^{i}$ for $c=0$ and $c=1$, therefore we present the values of these sums first. We have

$$
\sum_{i=0}^{h} n^{i}=\frac{n^{h+1}-1}{n-1} \text { and } \sum_{i=1}^{h} n^{i}=\frac{n\left(n^{h}-1\right)}{n-1} .
$$

Then

$$
(1-n) \sum_{i=0}^{h} i n^{i}=n+n^{2}+n^{3}+\ldots+n^{h}-h n^{h+1}=\frac{n\left(1-n^{h}\right)}{1-n}-h n^{h+1}
$$

and

$$
\sum_{i=0}^{h} i n^{i}=\frac{n\left(1-n^{h}\right)}{(1-n)^{2}}+\frac{h n^{h+1}}{n-1}=\frac{h n^{h+2}-(h+1) n^{h+1}+n}{(n-1)^{2}} .
$$

Since $(n-1)^{2} \sum_{i=0}^{h} i^{2} n^{i}$

$$
\begin{aligned}
& =n+2 n^{2}+2 n^{3}+2 n^{4}+\ldots+2 n^{h}-\left(h^{2}+2 h-1\right) n^{h+1}+h^{2} n^{h+2} \\
& =n+2 n^{2}\left(1+n+n^{2}+\ldots+n^{h-2}\right)-\left(h^{2}+2 h-1\right) n^{h+1}+h^{2} n^{h+2} \\
& =\frac{2 n^{2}\left(n^{h-1}-1\right)}{n-1}+n-\left(h^{2}+2 h-1\right) n^{h+1}+h^{2} n^{h+2},
\end{aligned}
$$


we obtain

$$
\begin{aligned}
\sum_{i=0}^{h} i^{2} n^{i} & =\frac{2 n^{h+1}-2 n^{2}}{(n-1)^{3}}+\frac{h^{2} n^{h+2}-\left(h^{2}+2 h-1\right) n^{h+1}+n}{(n-1)^{2}} \\
& =\frac{h^{2} n^{h+3}-\left(2 h^{2}+2 h-1\right) n^{h+2}+(h+1)^{2} n^{h+1}-n^{2}-n}{(n-1)^{3}} .
\end{aligned}
$$

Note that $\sum_{i=0}^{h} i n^{i}=\sum_{i=1}^{h} i n^{i}$ and $\sum_{i=0}^{h} i^{2} n^{i}=\sum_{i=1}^{h} i^{2} n^{i}$.

All the theorems presented in this paper hold for $h \geq 1$ and $0 \leq p \leq$ $n-2$, where at least one of the integers $p$ and $n$ is even. For $a \in \mathbf{R}$, we study the general eccentricity index $E I_{a}$ of networks in $T_{n, h}^{p}$.

Theorem 1. Let $N$ be any network in $T_{n, h}^{p}$. Then

$$
E I_{a}(N)=\sum_{i=0}^{h} n^{i}(h+i)^{a}
$$

Proof. In $N$, the number of vertices of $S_{i}$ is $n^{i}$, where $i=0,1,2, \ldots, h$. The eccentricity of each vertex in $S_{i}$ is $h+i$. Therefore

$$
\begin{aligned}
E I_{a}(N) & =\sum_{u \in S_{0}} h^{a}+\sum_{u \in S_{1}}(h+1)^{a}+\sum_{u \in S_{2}}(h+2)^{a}+\ldots+\sum_{u \in S_{h}}(2 h)^{a} \\
& =n^{0} h^{a}+n^{1}(h+1)^{a}+n^{2}(h+2)^{a}+\ldots+n^{h}(2 h)^{a} \\
& =\sum_{i=0}^{h} n^{i}(h+i)^{a}
\end{aligned}
$$

The total eccentricity index $E I_{1}$ of any biological network from the set $T_{n, h}^{p}$ is studied in Theorem 2 .

Theorem 2. Let $N$ be any network in $T_{n, h}^{p}$. Then

$$
E I_{1}(N)=\frac{2 h n^{h+2}-(2 h+1) n^{h+1}-h n+n+h}{(n-1)^{2}} .
$$


Proof. From Theorem 1, we obtain

$$
E I_{1}(N)=\sum_{i=0}^{h} n^{i}(h+i) .
$$

Thus

$$
\begin{aligned}
E I_{1}(N) & =h \sum_{i=0}^{h} n^{i}+\sum_{i=0}^{h} i n^{i} \\
& =\frac{h n^{h+1}-h}{n-1}+\frac{h n^{h+2}-(h+1) n^{h+1}+n}{(n-1)^{2}} \\
& =\frac{2 h n^{h+2}-(2 h+1) n^{h+1}-h n+n+h}{(n-1)^{2}} .
\end{aligned}
$$

The first Zagreb eccentricity index $\xi_{1}\left(=E I_{2}\right)$ is investigated in Theorem 3.

Theorem 3. Let $N$ be any network in $T_{n, h}^{p}$. Then

$$
\begin{aligned}
\xi_{1}(N)= & \frac{4 h^{2} n^{h+3}-\left(6 h^{2}+6 h-1\right) n^{h+2}+(2 h+1)^{2} n^{h+1}-(h-1)^{2} n^{2}}{(n-1)^{3}} \\
& +\frac{\left(2 h^{2}-2 h-1\right) n-h^{2}}{(n-1)^{3}} .
\end{aligned}
$$

Proof. From Theorem 1, we obtain

$$
E I_{2}(N)=\xi_{1}(N)=\sum_{i=0}^{h} n^{i}(h+i)^{2} .
$$

Thus

$$
\begin{aligned}
\xi_{1}(N) & =\sum_{i=0}^{h} n^{i}\left(h^{2}+2 h i+i^{2}\right) \\
& =h^{2} \sum_{i=0}^{h} n^{i}+2 h \sum_{i=0}^{h} i n^{i}+\sum_{i=0}^{h} i^{2} n^{i}
\end{aligned}
$$




$$
\begin{aligned}
= & h^{2}\left(\frac{n^{h+1}-1}{n-1}\right)+2 h\left(\frac{h n^{h+2}-(h+1) n^{h+1}+n}{(n-1)^{2}}\right) \\
& +\frac{h^{2} n^{h+3}-\left(2 h^{2}+2 h-1\right) n^{h+2}+(h+1)^{2} n^{h+1}-n^{2}-n}{(n-1)^{3}} \\
= & \frac{4 h^{2} n^{h+3}-\left(6 h^{2}+6 h-1\right) n^{h+2}+(2 h+1)^{2} n^{h+1}-(h-1)^{2} n^{2}}{(n-1)^{3}} \\
& +\frac{\left(2 h^{2}-2 h-1\right) n-h^{2}}{(n-1)^{3}} .
\end{aligned}
$$

Let us introduce the general invariant

$$
I(G)=\sum_{u v \in E(G)} f\left(\operatorname{ecc}_{N}(u), e c c_{N}(v)\right),
$$

with $f\left(e c c_{N}(u), e c c_{N}(v)\right)$ being a function of $e c c_{N}(u)$ and $e c c_{N}(v)$ where $f\left(\operatorname{ecc}_{N}(u), e c c_{N}(v)\right)=f\left(e c c_{N}(v), e c c_{N}(u)\right)$.

The second Zagreb eccentricity index is obtained if $f\left(\operatorname{ecc}_{N}(u), e c c_{N}(v)\right)=$ $\operatorname{ecc}_{N}(u) e c c_{N}(v)$. The modified Zagreb eccentricity index is obtained if $f\left(e c c_{N}(u), e c c_{N}(v)\right)=e c c_{N}(u)+e c c_{N}(v)$.

Theorem 4. Let $N$ be any network in $T_{n, h}^{p}$. Then

$$
I(N)=\sum_{i=1}^{h}\left[n^{i} f(h+i-1, h+i)+\frac{p n^{i}}{2} f(h+i, h+i)\right] .
$$

Proof. The number of edges in $N$ is

$$
\left(n+n^{2}+\ldots+n^{h}\right)+\left(\frac{p n}{2}+\frac{p n^{2}}{2}+\ldots+\frac{p n^{h}}{2}\right) .
$$

Let

$$
E_{j, l}=\left\{u v \in E(N) \mid u \in S_{j}, v \in S_{l}\right\} .
$$

We have

$$
E(N)=\left(E_{1,1} \cup E_{2,2} \cup \ldots \cup E_{h, h}\right) \cup\left(E_{0,1} \cup E_{1,2} \cup \ldots \cup E_{h-1, h}\right) .
$$


We get

$$
\left|E_{0,1}\right|=n^{1},\left|E_{1,2}\right|=n^{2}, \ldots,\left|E_{h-1, h}\right|=n^{h}
$$

and

$$
\left|E_{1,1}\right|=\frac{p n}{2},\left|E_{2,2}\right|=\frac{p n^{2}}{2}, \ldots,\left|E_{h, h}\right|=\frac{p n^{h}}{2} .
$$

Each vertex in $S_{i}$ is of eccentricity $h+i$, where $i=0,1,2, \ldots, h$, therefore

$$
\begin{aligned}
I(N)= & \sum_{u v \in E(N)} f\left(\operatorname{ecc}_{N}(u), e c c_{N}(v)\right) \\
= & \sum_{u v \in E_{0,1}} f(h, h+1)+\sum_{u v \in E_{1,2}} f(h+1, h+2)+\ldots \\
& +\sum_{u v \in E_{h-1, h}} f(2 h-1,2 h) \\
& +\sum_{u v \in E_{1,1}} f(h+1, h+1)+\sum_{u v \in E_{2,2}} f(h+2, h+2)+\ldots \\
& +\sum_{u v \in E_{h, h}} f(2 h, 2 h) \\
= & n \cdot f(h, h+1)+n^{2} f(h+1, h+2)+\ldots+n^{h} f(2 h-1,2 h) \\
& +\frac{p n}{2} f(h+1, h+1)+\frac{p n^{2}}{2} f(h+2, h+2)+\ldots+\frac{p n^{h}}{2} f(2 h, 2 h) \\
= & \sum_{i=1}^{h} n^{i} f(h+i-1, h+i)+\sum_{i=1}^{h} \frac{p n^{i}}{2} f(h+i, h+i) .
\end{aligned}
$$

We use Theorem 4 to study the $\xi_{1}^{\prime}$ and $\xi_{2}$ indices of biological networks.

Theorem 5. Let $N$ be any network in $T_{n, h}^{p}$. Then the modified Zagreb eccentricity index

$$
\begin{aligned}
\xi_{1}^{\prime}(N)= & \frac{(2 p h+4 h-1) n^{h+2}-(2 p h+4 h+p+1) n^{h+1}-(p h+2 h-1) n^{2}}{(n-1)^{2}} \\
& +\frac{(p h+2 h+p+1) n}{(n-1)^{2}}
\end{aligned}
$$

and the second Zagreb eccentricity index 


$$
\begin{aligned}
\xi_{2}(N)= & h\left(\frac{p h}{2}+h-1\right) \frac{n^{h+1}-n}{n-1}+(p h+2 h-1) \frac{h n^{h+2}-(h+1) n^{h+1}+n}{(n-1)^{2}} \\
& +\left(\frac{p}{2}+1\right)\left[\frac{h^{2} n^{h+3}-\left(2 h^{2}+2 h-1\right) n^{h+2}+(h+1)^{2} n^{h+1}-n^{2}-n}{(n-1)^{3}}\right] .
\end{aligned}
$$

Proof. We have $f\left(\operatorname{ecc}_{N}(u), \operatorname{ecc}_{N}(v)\right)=e c c_{N}(u)+e c c_{N}(v)$ in the case of $\xi_{1}^{\prime}$ index. Thus $f(h+i-1, h+i)=2 h+2 i-1$ and $f(h+i, h+i)=2 h+2 i$.

From Theorem 4, we get

$$
\begin{aligned}
\xi_{1}^{\prime}(N)= & \sum_{i=1}^{h}\left[n^{i}(2 h+2 i-1)+\frac{p n^{i}}{2}(2 h+2 i)\right] \\
= & (p h+2 h-1) \sum_{i=1}^{h} n^{i}+(p+2) \sum_{i=1}^{h} i n^{i} \\
= & (p h+2 h-1) \frac{n\left(n^{h}-1\right)}{n-1}+(p+2) \frac{h n^{h+2}-(h+1) n^{h+1}+n}{(n-1)^{2}} \\
= & \frac{(2 p h+4 h-1) n^{h+2}-(2 p h+4 h+p+1) n^{h+1}-(p h+2 h-1) n^{2}}{(n-1)^{2}} \\
& +\frac{(p h+2 h+p+1) n}{(n-1)^{2}} .
\end{aligned}
$$

We have $f\left(\operatorname{ecc}_{N}(u), \operatorname{ecc}_{N}(v)\right)=e c c_{N}(u) e c c_{N}(v)$ in the case of $\xi_{2}$ index. Thus $f(h+i-1, h+i)=(h+i-1)(h+i)=h^{2}-h+2 h i-i+i^{2}$ and $f(h+i, h+i)=(h+i)(h+i)=h^{2}+2 h i+i^{2}$. From Theorem 4, we get

$$
\begin{aligned}
\xi_{2}(N)= & \sum_{i=1}^{h}\left[n^{i}\left(h^{2}-h+2 h i-i+i^{2}\right)+\frac{p n^{i}}{2}\left(h^{2}+2 h i+i^{2}\right)\right] \\
= & h\left(\frac{p h}{2}+h-1\right) \sum_{i=1}^{h} n^{i}+(p h+2 h-1) \sum_{i=1}^{h} i n^{i}+\left(\frac{p}{2}+1\right) \sum_{i=1}^{h} i^{2} n^{i} \\
= & h\left(\frac{p h}{2}+h-1\right) \frac{n^{h+1}-n}{n-1}+(p h+2 h-1) \frac{h n^{h+2}-(h+1) n^{h+1}+n}{(n-1)^{2}} \\
& +\left(\frac{p}{2}+1\right)\left[\frac{h^{2} n^{h+3}-\left(2 h^{2}+2 h-1\right) n^{h+2}+(h+1)^{2} n^{h+1}-n^{2}-n}{(n-1)^{3}}\right] .
\end{aligned}
$$


In Corollary 1, we consider the situation when a person having a virus is in contact with one other person who got the virus from someone else. So $p=1$. See Figure 4 for the case in which a person transmits the virus to 4 other people, so $n=4$.

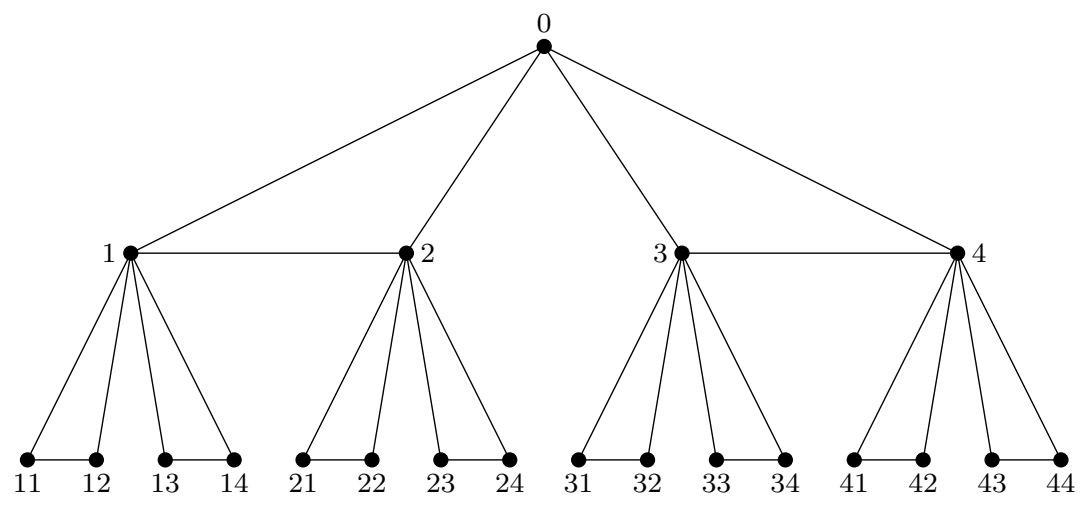

Figure 4: One of the network in $T_{n, h}^{p}$ for $p=1, n=4$ and $h=2$.

Corollary 1. Let $N$ be any network in $T_{n, h}^{1}$, where $n \geq 4$ is even and $h \geq 1$. Then the modified Zagreb eccentricity index

$$
\xi_{1}^{\prime}(N)=\frac{(6 h-1) n^{h+2}-(6 h+2) n^{h+1}-(3 h-1) n^{2}+(3 h+2) n}{(n-1)^{2}}
$$

and the second Zagreb eccentricity index

$$
\begin{aligned}
\xi_{2}(N)= & \frac{4 h(3 h-1) n^{h+3}-\left(24 h^{2}+4 h-5\right) n^{h+2}+(2 h+1)(6 h+1) n^{h+1}}{2(n-1)^{3}} \\
& +\frac{h(2-3 h) n^{3}+\left(6 h^{2}+2 h-5\right) n^{2}-\left(3 h^{2}+4 h+1\right) n}{2(n-1)^{3}} .
\end{aligned}
$$

Proof. We use $p=1$ in Theorem 5 to obtain

$$
\xi_{1}^{\prime}(N)=\frac{(6 h-1) n^{h+2}-(6 h+2) n^{h+1}-(3 h-1) n^{2}+(3 h+2) n}{(n-1)^{2}}
$$

and 


$$
\begin{aligned}
\xi_{2}(N)= & h\left(\frac{3 h-2}{2}\right) \frac{n^{h+1}-n}{n-1}+(3 h-1) \frac{h n^{h+2}-(h+1) n^{h+1}+n}{(n-1)^{2}} \\
& +\frac{3}{2}\left[\frac{h^{2} n^{h+3}-\left(2 h^{2}+2 h-1\right) n^{h+2}+(h+1)^{2} n^{h+1}-n^{2}-n}{(n-1)^{3}}\right] \\
= & \frac{4 h(3 h-1) n^{h+3}-\left(24 h^{2}+4 h-5\right) n^{h+2}+(2 h+1)(6 h+1) n^{h+1}}{2(n-1)^{3}} \\
& +\frac{h(2-3 h) n^{3}+\left(6 h^{2}+2 h-5\right) n^{2}-\left(3 h^{2}+4 h+1\right) n}{2(n-1)^{3}} .
\end{aligned}
$$

It is easy to check that for example for $p=1$ and $h=2$, we get $\xi_{1}^{\prime}(N)=11 n^{2}+8 n$ and $\xi_{2}(N)=20 n^{2}+\frac{21}{2} n$.

In Corollary 2, we consider the case when a person having the virus is not in contact with people who got the virus from someone else. So $p=0$. For each $n \geq 2$ and $h \geq 1$, the only tree in the set $T_{n, h}^{0}$ is the complete $n$-ary tree of height $h$; see Figure 5.

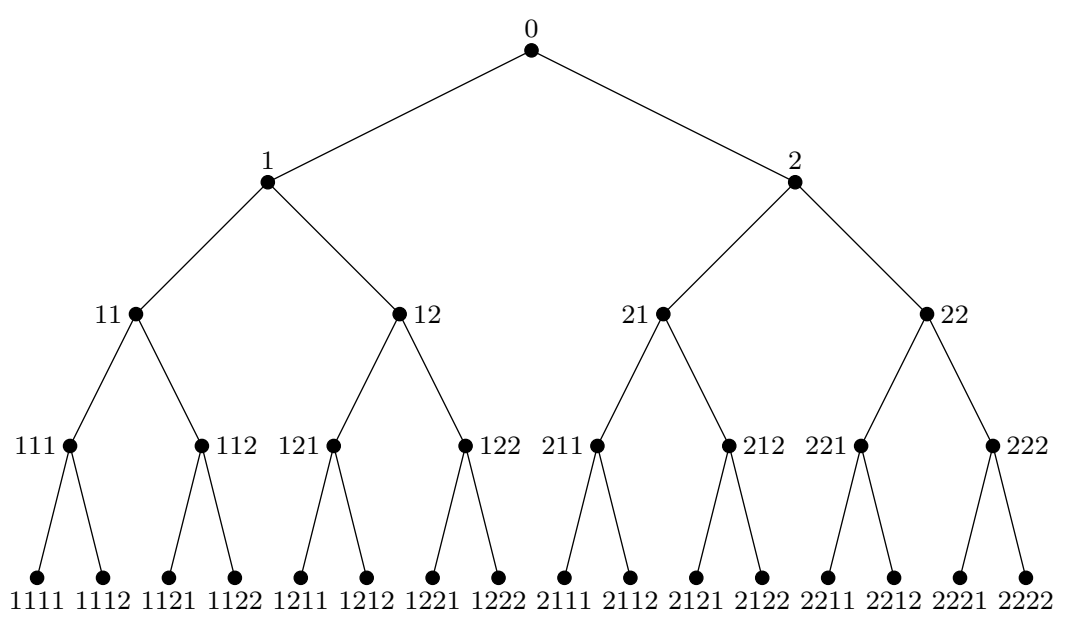

Figure 5: Complete $n$-ary tree $T_{n, h}$ for $n=2$ and $h=4$.

Corollary 2. For the complete $n$-ary tree $T_{n, h}$ of height $h$, where $n \geq 2$ and $h \geq 1$, we get

$$
\xi_{1}^{\prime}\left(T_{n, h}\right)=\frac{(4 h-1) n^{h+2}-(4 h+1) n^{h+1}-(2 h-1) n^{2}+(2 h+1) n}{(n-1)^{2}}
$$


and

$$
\begin{aligned}
\xi_{2}\left(T_{n, h}\right)= & \frac{2 h(2 h-1) n^{h+3}-\left(8 h^{2}-2\right) n^{h+2}+2 h(2 h+1) n^{h+1}}{(n-1)^{3}} \\
& -\frac{h(h-1) n^{3}+2\left(h^{2}-1\right) n^{2}-h(h+1) n}{(n-1)^{3}} .
\end{aligned}
$$

Proof. We use $p=0$ in Theorem 5 to obtain the $\xi_{1}^{\prime}$ index of $T_{n, h} \in T_{n, h}^{0}$,

$$
\xi_{1}^{\prime}\left(T_{n, h}\right)=\frac{(4 h-1) n^{h+2}-(4 h+1) n^{h+1}-(2 h-1) n^{2}+(2 h+1) n}{(n-1)^{2}}
$$

and

$$
\begin{aligned}
\xi_{2}\left(T_{n, h}\right)= & h(h-1) \frac{n^{h+1}-n}{n-1}+(2 h-1) \frac{h n^{h+2}-(h+1) n^{h+1}+n}{(n-1)^{2}} \\
& +\frac{h^{2} n^{h+3}-\left(2 h^{2}+2 h-1\right) n^{h+2}+(h+1)^{2} n^{h+1}-n^{2}-n}{(n-1)^{3}} \\
= & \frac{2 h(2 h-1) n^{h+3}-\left(8 h^{2}-2\right) n^{h+2}+2 h(2 h+1) n^{h+1}}{(n-1)^{3}} \\
& -\frac{h(h-1) n^{3}+2\left(h^{2}-1\right) n^{2}-h(h+1) n}{(n-1)^{3}} .
\end{aligned}
$$

It is easy to check that for example for $h=2$, we get $\xi_{1}^{\prime}\left(T_{n, h}\right)=7 n^{2}+5 n$ and $\xi_{2}\left(T_{n, h}\right)=12 n^{2}+6 n$.

\section{Conclusion}

Gao et al. [4] and Imran et al. [7] investigated biological networks assuming that a virus is transmitted from one person to two other people, and a person having the virus is in contact with exactly one other person who got the virus from someone else. We extended research in this area by studying biological networks in which a person transmits a virus to $n$ other people, where $n \geq 2$, and a person having the virus is in contact with $p$ other people $(0 \leq p \leq n-2)$ who got the virus from someone else. We obtain eccentricity based indices of these biological networks. 


\section{Acknowledgment}

This work is based on the research supported by the National Research Foundation of South Africa (Grant Number 129252).

\section{References}

[1] M. Cancan, S. Ediz, A. Q. Baig, and W. Khalid, "M-Polynomial and topological indices of benzene ring embedded in p-type surface network", Proyecciones (Antofagasta), vol. 39, no. 4, pp. 749-768, 2020, doi: 10.22199/ issn.0717-6279-2020-04-0047

[2] M. Cancan, M. Hussain, and H. Ahmad, "Distance and eccentricity based polynomials and indices of $\mathrm{m}$-level Wheel graph", Proyecciones (Antofagasta), vol. 39, no. 4, pp. 869-885, 2020, doi: 10.22199/issn. 0717-62 79-2020-04-0054

[3] R. Farooq and M. A. Malik, "On some eccentricity based topological indices of nanostar dendrimers", Optoelectronics and Advanced Materials, Rapid Communications, vol. 9, no. 5-6, pp. 842-849, 2015.

[4] W. Gao, H. Wu, M. K. Siddiqui, and A. Q. Baig, "Study of biological networks using graph theory", Saudi Journal of Biological Sciences, vol. 25, no. 6, pp. 1212-1219, 2018, doi: 10.1016/j.sjbs.2017.11.022

[5] M. Ghorbani and M. Hosseinzadeh, "A new version of Zagreb indices", Filomat, vol. 26, no. 1, pp. 93-100, 2012, doi: 10.2298/ FIL1201093G

[6] M. Imran, A. A. E. Abunamous, D. Adi, S. H. Rafique, A. Q. Baig, and M. R. Farahani, "Eccentricity based topological indices of honeycomb networks," Journal of Discrete Mathematical Sciences and Cryptography, vol. 22, no. 7, pp. 1199-1213, 2019, doi: 10.1080/ 09720529.2019 .1691326

[7] M. Imran, M. A. Iqbal, Y. Liu, A. Q. Baig, W. Khalid, and M. A. Zaighum, "Computing Eccentricity-Based Topological Indices of 2-Power Interconnection Networks", Journal of Chemistry, vol. 2020, pp. 1-7, 2020, doi: $10.1155 / 2020 / 3794592$

[8] M. Imran, M. Siddiqui, A. Abunamous, D. Adi, S. Rafique, and A. Baig, "Eccentricity Based Topological Indices of an Oxide Network", Mathematics, vol. 6, no. 7, p. 126, 2018, doi: 10.3390/math6070126 
[9] A. J. M. Khalaf, A. Javed, M. K. Jamil, M. Alaeiyan, and M. R. Farahani, "Topological properties of four types of porphyrin dendrimers", Proyecciones (Antofagasta), vol. 39, no. 4, pp. 979-993, 2020, doi: 10.22199/ issn.0717-6279-2020-04-0061

[10] J.-B. Liu, H. Shaker, I. Nadeem, and M. R. Farahani, "Eccentric Connectivity Index of t-Polyacenic Nanotubes", Advances in Materials Science and Engineering, vol. 2019, pp. 1-9, 2019, doi: 10.1155/2019/ 9062535

[11] D. Vukičević, and A, Graovac, "Note on the comparison of the first and second normalized Zagreb eccentricity indices", Acta Chimica Slovenica, vol. 57, no. 3, pp. 524-528, 2010.

\author{
Tomáš Vetrík \\ Department of Mathematics and Applied Mathematics \\ University of the Free State \\ Bloemfontein \\ South Africa \\ e-mail:vetrikt@ufs.ac.za
}

